\title{
Calibration of Modulation Transfer Function of Surface Profilometers with 1D and 2D Binary Pseudo-random Array Standards
}

\author{
Valeriy V. Yashchuk, ${ }^{\mathrm{a} *}$ Wayne R. McKinney, ${ }^{\mathrm{a}}$ and Peter Z. Takacs ${ }^{\mathrm{b}}$ \\ ${ }^{a)}$ Lawrence Berkeley National Laboratory, Berkeley, CA 94720, USA \\ ${ }^{b)}$ Brookhaven National Laboratory, Upton, New York, 11973, USA \\ *) vvyashchuk@lbl.gov
}

\begin{abstract}
We suggest and describe the use of a binary pseudo-random grating as a standard test surface for calibration of the modulation transfer function of microscopes. Results from calibration of a Micromap $^{\mathrm{TM}}$-570 interferometric microscope are presented.

(C)2008 Optical Society of America

OCIS codes: (120.4800) Optical standards and testing; (120.6660) Surface measurements, roughness
\end{abstract}

\section{Introduction}

Knowledge of the modulation transfer function (MTF) of a microscope is absolutely necessary to convert the measured height distribution of a surface undergoing metrology into an accurate power spectral density (PSD) distribution. We suggest and describe the use of a binary pseudo-random (BPR) grating as a standard test surface for measurement of the MTF of microscopes [1-3]. For an 'ideal' microscope with an MTF function independent of spatial frequency out to the Nyquist frequency of the detector array with zero response at higher spatial frequencies, a BPR grating would produce a flat 1D PSD spectrum, independent of spatial frequency. For a 'real' instrument, the MTF is found as the square root of the ratio of the PSD spectrum measured with the BPR grating to the 'ideal', spatial frequency independent, PSD spectrum. We also present the results from a measurement of the MTF of a Micromap ${ }^{\mathrm{TM}}-570$ interferometric microscope demonstrating the effectiveness of the calibration method. The measured PSD distributions provide a framework for connecting surface roughness with three-dimensional calculations of scattering of light by the optical surfaces [4].

A straightforward transformation of the measured 2D area distribution of the residual surface heights into a 2D PSD distribution almost always provides spectra with distortion caused by the unknown spatial frequency response of the instrument. The response is characterized by MTF, which is defined by the spatial frequency bandwidth of the instrument. The MTF contains contributions from the instrument's optical system, detector, signal processing, software algorithm, and environmental factors. Generally, these contributions are difficult to account for separately. The instrumental MTF can be evaluated by comparing a measured PSD distribution of a known test surface with the corresponding ideal numerically simulated PSD. The square root of the ratio of the measured and simulated PSD distributions gives the MTF of the instrument. The binary pseudo-random grating (BPRG) described here provides an effective test surface to fulfill this calibration need.

\section{Binary Pseudo-random Distributions}

The BPRG as we determine it here is a set of rectangular grooves (with a binary height distribution) pseudo-randomly distributed over a uniform grid with an elementary pitch equal to the width of the smallest groove. The term 'pseudo-random' depicts that the distribution is specially generated to possess a property of randomness in the mathematically strong sense. As it is shown below, the inherent PSD spectrum of such a grating is independent of spatial frequency (white-noise-like). Therefore, any deviation of a PSD spectrum measured with a real instrument from a white-noise-like spectrum would be a measure of the instrumental MTF.

The analytical method we used to generate a MLPRS (modulo-two) of odd length $N=2^{n}-1$, where $n$ is an integer, is described in Ref. [5]. In this article, all base ten values for the recursion coefficients (RC) which can be used for the generation of MLPRS are presented and the use of a particular RC to generate a pseudo-random sequence is explained. Based on the procedure described in Ref. [5], we generate a BPR sequence of $N=4095$ elements obtained (see Ref. [5], Table III) with sequence generator $n=12$ and recursion coefficient $M=83$ (base 10 value). 


\section{Binary Pseudo-random Grating for Calibration of Modulation Transfer Function}

A few gratings according to the generated BPR sequence were fabricated using a conventional lithographical process. The gratings were etched into a silicon (110) wafer using anisotropic etching in a $\mathrm{KOH}$ solution. The fundamental feature width of the gratings is $2.5 \mu \mathrm{m}$. The etch depth was measured with a calibrated atomic force microscope to be approximately 110, 440, and $700 \mathrm{~nm}$ for the particular gratings.

The PSD spectrum of the complete ideal BPRG function is a white-noise-like straight line with no fluctuations and with amplitude of 0.5. The amplitude corresponds to an expectation value based on the duty cycle of approximately 0.5 of the maximum-length pseudo-random sequence used in the construction. For real experimental arrangements, when an instrument with finite detector pixel size is used, one can not expect the spacing of the grating projected onto the detector to line up exactly with the boundaries of each detector pixel. The speckle noise thus produced is significantly reduced by averaging the spectrum of the shorter subsets of the main pattern, each shifted successively by a significant portion of the subset length. However, the success of the MTF calibration with the BPRG is still ensured by the deterministic character of the pseudo-random sequence used to fabricate the BPRG test surface and, therefore, the possibility to precisely simulate the PSD spectrum inherent for a certain part of the grating.

\section{Calibration of Micromap- $570^{\mathrm{TM}}$ Interferometric Microscope}

In the case of the Micromap- $570^{\mathrm{TM}}$, the instrumental MTF is seen as high frequency roll-off of the measured PSD spectrum. The roll-off is the result of two primary MTF effects: the transfer function for a diffraction-limited objective with incoherent illumination and the instrumental MTF associated with sampling with finite pixels. Both effects can be accounted for analytically. Using the BPRG we are able to experimentally find the instrumental MTF and correct the measured PSD spectra for the MTF by experimentally finding the parameters for the analytical expressions, specific for the instrument - Fig. 1 .

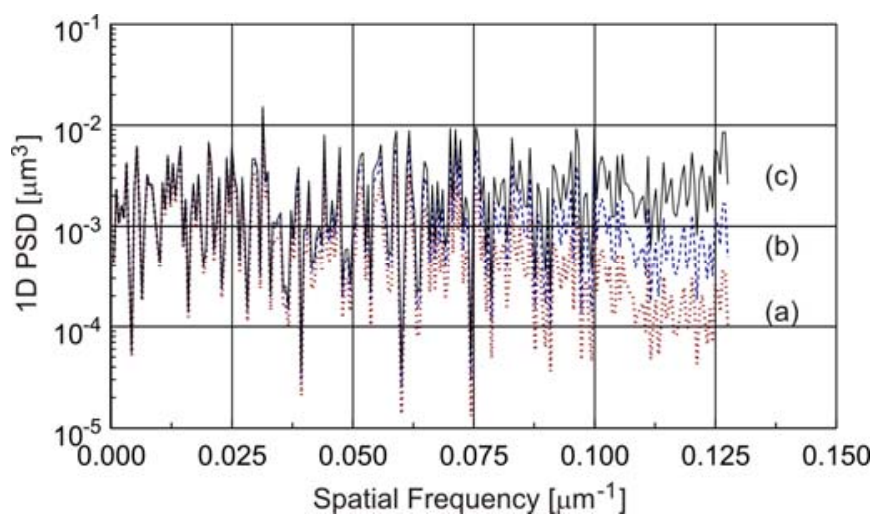

Fig. 1. 1D PSD spectrum of the prototype BPRG with 4095 total number of elements with $2.5-\mu \mathrm{m}$ pitch measured with the Micromap $^{\mathrm{TM}}$-570 microscope with 2.5× objective: a) (lower, red) average uncorrected raw PSD curve; b) (middle, blue) corrected to account for MTF due to the objective lens aperture, and c) additionally corrected for the MTF of the finite pixel width.

The choice of a binary pseudo-random sequence for the test grating has two major advantages compared with random 1D surfaces built based on sequences obtained with random number generators or white noise sources, e.g., with a grinding process. Both advantages relate to the requirement of ease of specification and reproducibility of the test surface when used as a certified standard. First, a binary height distribution with two normalized heights, ' 1 ' and ' 0 ', can be easily specified for a number of production processes, e.g., lithography. The absolute value of the height would be determined based on requirements for a particular application, such as the range of measurable heights of the instruments. Possible perturbations of the shape of the rectangular grooves of a BPR grating would just lead to a slight change of overall amplitude of the flat PSD spectrum without any noticeable perturbation of its spatial frequency dependence (flat) for frequencies lower than the frequency corresponding to the characteristic size of a unit groove. Second, a pseudo-random sequence has spectral characteristics that are mathematically rigorous, reproducible and amenable to simulation, allowing one to deterministically construct a maximum-length random sequence with an ideal ('one-bit' wide) autocorrelation function optimal for a particular instrument. 


\section{Discussion}

A distinguishing property of the BPR grating is that its PSD spectrum is a result of the distribution of the grooves, rather than the groove shape. This determines a low sensitivity of the BPRG PSD spectrum to the shape perturbation of a groove, which would be seen only at frequencies significantly higher than the Nyquist frequency of the instrument. In any case, if the perturbation is random, it does not change the inherent random (white-noise-like) character of the BPRG PSD spectrum. Moreover, the overall magnitude of the BPRG PSD spectrum is determined by the depth of the grooves. Therefore, for a reasonably designed BPRG standard, the contribution of the roughness of the grating surface can be easily made to be insignificant.

The deterministic character of the BPR grating allows precise simulation of the theoretical (ideal) PSD spectrum of the standard and comparison of it to an experimentally measured spectrum. But for some applications, the theoretical spectrum can be approximated with an ideal (without variation) white-noiselike spectrum. In this case, the amplitude of fluctuations of the measured PSD spectrum can be significantly decreased by averaging the PSD spectra measured at random shifts of the BPR grating with respect to the field of view of the instrument.

Even though in this summary we discuss the design and properties of a 1D BPR grating, the suggested approach has been extended to the two-dimensional (2D) case, when a test surface with a binary pseudorandom 2D pattern were designed. Such a test surface has the same advantages (a mathematically deterministic character and ease of specification and reproduction), in comparison to a 2D gray random target constructed with a generator of random numbers. An additional advantage of the 2D pseudo-random PSD standard would be the possibility for a direct 2D calibration of the instrumental MTF.

\section{Acknowledgements}

The authors are grateful to Rossana Cambie, John Warren, Don Elliott, Abdel Isakovic, and Rolf Beuttenmuller for fabrication of the BPRG test surface and Farhad Salmassi and Dmitry Voronov for measurements with an atomic force microscope. The Advanced Light Source is supported by the Director, Office of Science, Office of Basic Energy Sciences, Material Science Division, of the U.S. Department of Energy under Contract No. DE-AC02-05CH11231 at Lawrence Berkeley National Laboratory. This manuscript has been authored in part by Brookhaven Science Associates, LLC under Contract No. DEAC02-98CH10886 with the U.S. Department of Energy.

\section{References}

[1] V. V. Yashchuk, W. R. McKinney, P.Z. Takacs, Binary Pseudorandom Grating as a Standard Test Surface for measurement of Modulation Transfer Function of Interferometric Microscopes, Proc. of SPIE Vol. 6704, 670408 (2007)

[2] V. V. Yashchuk, W. R. McKinney, P.Z. Takacs, Binary Pseudo-random Grating Standard for Calibration of Surface Profilometers, Optical Engineering (July, 2008); accepted for publication.

[3] A patent application covering the described technology has been filed.

[4] W. R. McKinney, M. R. Howells, V. V. Yashchuk, Integration of the Two-dimensional Power Spectral Density into Specification for the X-ray Domain - Problems and Opportunities, this conference (Rochester, October, 2008).

[5] D. D. Koleske, and S. J. Sibener, Generation of pseudo-random sequence for use in cross-correlation modulation, Rev. Sci. Instrum. 63(8), 3852-5 (1992). 